\title{
Estimation of velocity vector angles using the directional cross-correlation method
}

\author{
Kortbek, Jacob; Jensen, Jørgen Arendt
}

Published in:

I E E E Transactions on Ultrasonics, Ferroelectrics and Frequency Control

Link to article, DOI:

10.1109/TUFFC.2006.144

Publication date:

2006

Document Version

Publisher's PDF, also known as Version of record

Link back to DTU Orbit

Citation (APA):

Kortbek, J., \& Jensen, J. A. (2006). Estimation of velocity vector angles using the directional cross-correlation method. I E E E Transactions on Ultrasonics, Ferroelectrics and Frequency Control, 53(11), 2036-2049. https://doi.org/10.1109/TUFFC.2006.144

\section{General rights}

Copyright and moral rights for the publications made accessible in the public portal are retained by the authors and/or other copyright owners and it is a condition of accessing publications that users recognise and abide by the legal requirements associated with these rights.

- Users may download and print one copy of any publication from the public portal for the purpose of private study or research.

- You may not further distribute the material or use it for any profit-making activity or commercial gain

- You may freely distribute the URL identifying the publication in the public portal 


\title{
Estimation of Velocity Vector Angles Using the Directional Cross-Correlation Method
}

\author{
Jacob Kortbek and Jørgen Arendt Jensen, Senior Member, IEEE
}

\begin{abstract}
A method for determining both velocity magnitude and angle in any direction is suggested. The method uses focusing along the velocity direction and crosscorrelation for finding the correct velocity magnitude. The angle is found from beamforming directional signals in a number of directions and then selecting the angle with the highest normalized correlation between directional signals. The approach is investigated using Field II simulations and data from the experimental ultrasound scanner RASMUS and a circulating flow rig with a parabolic flow having a peak velocity of $0.3 \mathrm{~m} / \mathrm{s}$. A $7-\mathrm{MHz}$ linear array transducer is used with a normal transmission of a focused ultrasound field. In the simulations the relative standard deviation of the velocity magnitude is between $0.7 \%$ and $7.7 \%$ for flow angles between $45^{\circ}$ and $90^{\circ}$. The study showed that angle estimation by directional beamforming can be estimated with a high precision. The angle estimation performance is highly dependent on the choice of the time $k_{\text {tprf }} \cdot T_{\text {prf }}$ (correlation time) between signals to correlate. One performance example is given with a fixed value of $\boldsymbol{k}_{\text {tprf }}$ for all flow angles. The angle estimation on measured data for flow at $60^{\circ}$ to $90^{\circ}$ yields a probability of valid estimates between $68 \%$ and $98 \%$. The optimal value of $\boldsymbol{k}_{\text {tprf }}$ for each flow angle is found from a parameter study; with these values, the performance on simulated data yields angle estimates with no outlier estimates and with standard deviations below $2^{\circ}$.
\end{abstract}

\section{INTRODUCTION}

$\mathrm{M}$ ODERN ultrasound systems can estimate the blood velocity in vivo in real time [1], [2]. This is done by acquiring ultrasound signals from the same direction a number of times, and then correlating the signals. The shift in phase as a function of time can be determined using an autocorrelation estimator and this yields the velocity [3]. A second approach is to determine the shift in position using the cross-correlation function; dividing with the time between emissions [4], [5] gives the velocity. Both approaches find only the shift along the ultrasound beam direction, and thus only the projected velocity along this direction. Velocities transverse to the beam are not detected, and velocities in different directions are not depicted correctly. There is, thus, a need for methods that can detect the correct velocity magnitude and direction as a function of spatial position.

Several authors have devised methods for estimating the correct velocity. Fox [6] suggested using two crossing beams to have two independent measurements. This, how-

Manuscript received November 28, 2005; accepted May 10, 2006.

The authors are with Ørsted•DTU, Technical University of Denmark, DK-2800 Lyngby, Denmark (e-mail: jk@oersted.dtu.dk).

Digital Object Identifier 10.1109/TUFFC.2006.144 ever, necessitates the use of a large aperture to obtain a sufficient angle between the beams to ensure a precise determination of the transverse component. Trahey and coworkers [7] suggested using speckle tracking over a region of the image to track the motion in any direction. This necessitates the use of fast beam formation to generate the large amounts of data. Introduction of a lateral oscillation has also been suggested so that the phase shift in the lateral directions also can be found using a modified autocorrelation method [8], [9]. Several other methods have been suggested [10], [11], but none have so far yielded a satisfactory performance to be commercially implemented.

This paper suggests a method for determining the velocity direction based on finding the motion along the velocity direction using cross-correlation. The velocity magnitude is determined here by focusing signals along the direction of the flow, as suggested in [12], [13]. This can be done if the correct direction is known. This is currently found by inspecting the B-mode image, and then using this angle in the beam formation. This is cumbersome and difficult to use, if the beam-to-flow angle changes throughout the image. Using the directional beamforming approach, angle estimation based on finding the direction of the maximum correlation is suggested in this paper, founded on the idea from [14]. Directional signals for a number of directions are beamformed, and the velocity and peak normalized correlation value are found. The direction with the highest correlation is then chosen as the angle estimate. The basics of the velocity estimation scheme is briefly described in Section II, and the angle estimation method is introduced in Section III. Investigations of the approach using simulations are given in Section IV-A and from measured data on a flow rig in Section IV-B.

\section{Directional Velocity Estimation}

This section gives a brief introduction to the principles from [12] of directional velocity estimation using focusing along the flow direction. The concept of spatial directional signals is introduced, and it is shown how the velocity can be estimated from these.

As described, the conventional cross-correlation method can estimate only the velocity component projected onto the direction of the ultrasound propagation, since the beam formation is done along this direction. In directional velocity estimation, the beam formation is done along the flow direction, and the correct velocity amplitude can, thus, be found also for a purely transverse direction. 


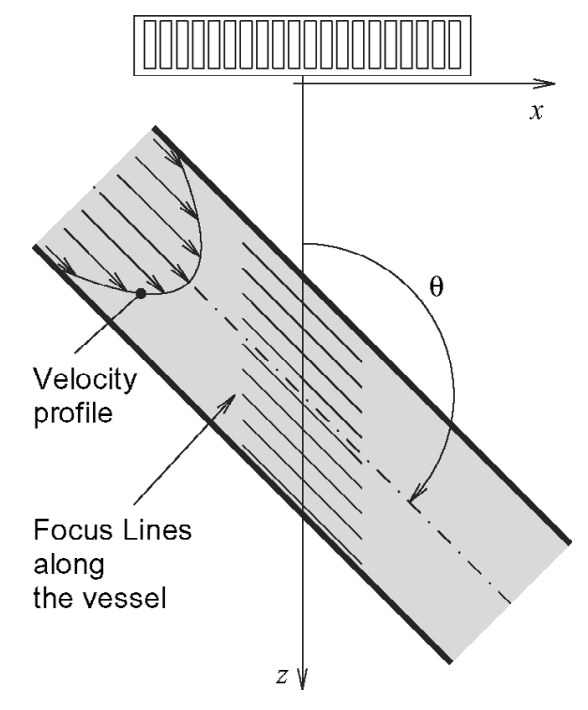

Fig. 1. Focus lines for constructing spatial directional signals.

The basic principle is to focus the responses received by the transducer along focus lines in the flow direction, which is referred to as directional signals in the spatial domain. In Fig. 1, a blood vessel and examples of focus lines are shown. The spatial signals for two consecutive emissions are then cross-correlated and the shift between them is found. This is a shift in spatial position of the scatterers, and dividing by the time between emissions, thus, directly gives the velocity magnitude. Just as for the conventional systems, the angle between the propagating direction of the emitted beam and the flow direction must be known before the beam formation can be done.

The directional signals are denoted $g\left(x^{\prime}\right)$, where $x^{\prime}$ is the $x$-coordinate in a rotated coordinate system aligned along the flow rather than along the ultrasound beam direction as shown in Fig. 2. Here the $x^{\prime}$-axis of the new coordinate system is parallel to the flow direction, and the origin of the coordinate system is placed at the center of the vessel at depth $Z_{\text {ves }}$. The original coordinate system $(x, z)$ has origin at the center of the transducer, the $x$-axis is parallel with the transducer, and the $z$-axis represents the depth. The $y$-axis represents the elevation plane, and this is the same for both coordinate systems. The relation between the rotated coordinate systems and the original coordinate system is given by

$$
\begin{aligned}
& x=\sin (\theta) \cdot x^{\prime}+\cos (\theta) \cdot z^{\prime} \\
& y=y^{\prime} \\
& z=-\cos (\theta) \cdot x^{\prime}+\sin (\theta) \cdot z^{\prime}+Z_{v e s}
\end{aligned}
$$

or in matrix notation,

$$
\left[\begin{array}{l}
x \\
z
\end{array}\right]=\mathbf{R}_{\mathbf{x z}} \cdot\left[\begin{array}{l}
x^{\prime} \\
z^{\prime}
\end{array}\right]+\left[\begin{array}{c}
0 \\
Z_{v e s}
\end{array}\right]
$$

where $\mathbf{R}_{\mathbf{x z}}$ is the rotation matrix:

$$
\mathbf{R}_{\mathbf{x z}}=\left[\begin{array}{cc}
\sin (\theta) & \cos (\theta) \\
-\cos (\theta) & \sin (\theta)
\end{array}\right]
$$

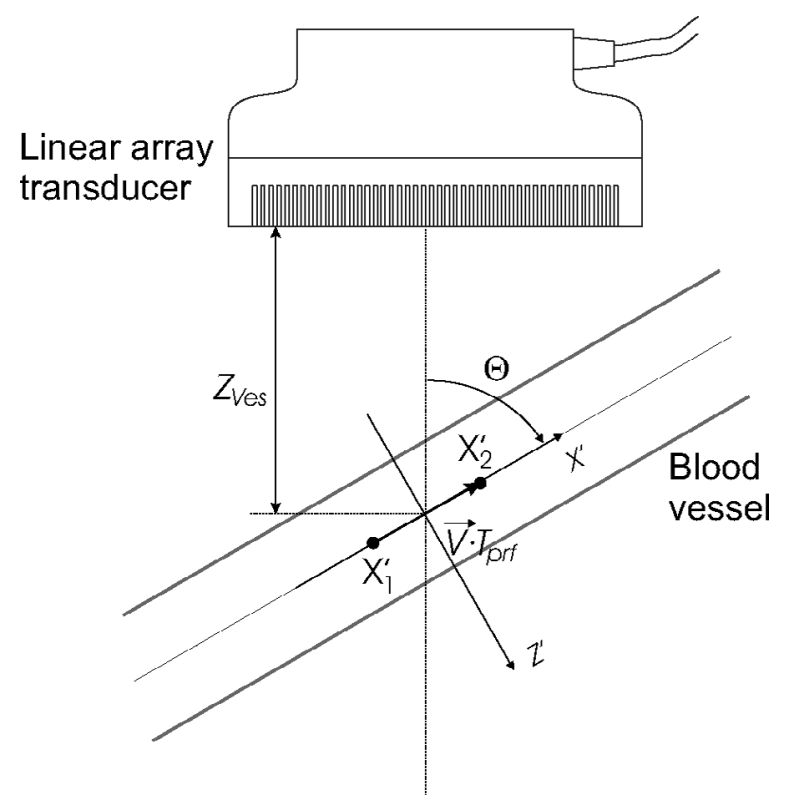

Fig. 2. Rotated coordinate system $\left(x^{\prime}, z^{\prime}\right)$ oriented along the flow direction, and with origin at the center of the vessel at a distance $Z_{v e s}$ from the transducer.

The angle $\theta$ is defined in Fig. 2. In the new coordinate system, the velocity vector is $\vec{v}=\left(v_{x^{\prime}}, 0,0\right)$, which has only one non-zero component, $v_{x^{\prime}}=|\vec{v}|$, provided the correct angle is used. The position of the scatterers can now be expressed through a scalar, where the first position is $x_{1}^{\prime}$, and the position after $T_{\mathrm{prf}}$ seconds is $x_{2}^{\prime}=x_{1}^{\prime}+v_{x^{\prime}} T_{\mathrm{prf}}$. The directional signals are used to find the velocity. The first signal is given by $g_{1}\left(x^{\prime}\right)$, and the second obtained $T_{\mathrm{prf}}$ seconds later is $g_{2}\left(x^{\prime}\right)$. The two signals are related by

$$
g_{2}\left(x^{\prime}\right)=g_{1}\left(x^{\prime}-v_{x^{\prime}} T_{\mathrm{prf}}\right) .
$$

Cross-correlating the two signals gives

$$
R_{12}\left(\xi_{x^{\prime}}\right)=\int_{X} g_{1}\left(x^{\prime}\right) g_{2}\left(x^{\prime}+\xi_{x^{\prime}}\right) d x^{\prime}
$$

where $X$ is the length of the directional signals. Using (4) the cross-correlation can be rewritten as

$$
\begin{aligned}
R_{12}\left(\xi_{x^{\prime}}\right) & =\int_{X} g_{1}\left(x^{\prime}\right) g_{1}\left(x^{\prime}-v_{x^{\prime}} T_{\mathrm{prf}}+\xi_{x^{\prime}}\right) d x^{\prime} \\
& =R_{11}\left(\xi_{x^{\prime}}-v_{x^{\prime}} T_{\mathrm{prf}}\right)
\end{aligned}
$$

which is the shifted autocorrelation $R_{11}\left(\xi_{x^{\prime}}\right)$ of $g_{1}\left(x^{\prime}\right)$, which has a global maximum at $\xi_{x^{\prime}}=v_{x^{\prime}} T_{\text {prf }}$. The maximum of the cross-correlation function

$$
\xi_{x_{\max }^{\prime}}=\underset{\xi_{x^{\prime}}}{\arg \max }\left\{R_{12}\left(\xi_{x^{\prime}}\right)\right\}
$$

is, thus, the shift in spatial position of the scatterers over the time interval $T_{\text {prf }}$. This makes it possible to calculate the velocity estimate along the flow direction, given by

$$
\hat{v}_{x^{\prime}}=\frac{\xi_{x_{\max }^{\prime}}}{T_{\mathrm{prf}}} .
$$


The cross-correlation can be improved by averaging over several estimates of $R_{12}$ under the assumption that the velocity of the scatterers can be considered constant for several pulses. A number of cross-correlations $N_{x c}$ can, thus, be used in the estimation. When applying averaging, $R_{12}\left(\xi_{x^{\prime}}\right)$ in (5) and (7) is substituted with

$$
R_{12}\left(\xi_{x^{\prime}}\right)=\sum_{i=1}^{N_{x c}} \mathcal{R}\left\{g_{i}\left(x^{\prime}, z^{\prime}\right), g_{i+1}\left(x^{\prime}, z^{\prime}\right)\right\}
$$

where $\mathcal{R}$ is the cross-correlation operator. The correct flow angle must be determined for this to work, and this is the topic of Section III.

The beam formation of the directional signals is performed as described in [12], [13]. The discrete directional signals are obtained by focusing signals received by the transducer elements in a set of points. These points are located on a straight line in any arbitrary direction and, e.g., aligned along the flow direction for velocity estimation. The focusing of any of these points is based on the total transmit-receive time-of-flight, and a sample of the directional signal corresponding to the point $\mathbf{r}_{\mathbf{x}}$ is calculated as

$$
g\left(\mathbf{r}_{\mathbf{x}}\right)=\sum_{j=1}^{N_{E}} s_{j}\left(t_{\mathbf{r}_{\mathbf{x}}}\right)=\sum_{j=1}^{N_{E}} s_{j}\left(\frac{\left|\mathbf{r}_{\mathbf{t}}-\mathbf{r}_{\mathbf{x}}\right|+\left|\mathbf{r}_{\mathbf{x}}-\mathbf{r}_{\mathbf{j}}\right|}{c}\right),
$$

where $N_{E}$ is the number of transducer elements, $s_{j}(t)$ is the received signal from transducer element $\mathbf{j}, t_{\mathbf{r}_{\mathbf{x}}}$ is the time instance at which to select a value from the signal $s_{j}(t), \mathbf{r}_{\mathbf{t}}$ is the position of the transmit source, and $\mathbf{r}_{\mathbf{j}}$ is the position of the $j$ 'th transducer element.

\section{Angle Estimation}

Conventional velocity estimation systems rely on knowledge of the angle between the flow vector and the direction of the emitted ultrasound beam, and traditionally this knowledge comes from the B-mode image. In the ideal situation, the vessel is rigid and straight without sudden geometric changes within the range of the B-mode image, or at least within the velocity estimation range. In this case, the beam-to-flow angle could be determined with satisfactory results from the B-mode image. Unfortunately, this is not a realistic case. The blood vessels are branching and curving, and typically no unique direction can be found for the whole image. This leads to a wrong correction value for the projected velocity component, and the consequence is an increase in bias and standard deviation. The approach also fails for angles close to $90^{\circ}$. This motivates the investigation of an automatic approach to angle estimation.

The spatial directional signals are obtained by focusing the received responses in points along the flow direction. Directional signals obtained from two consecutive acquired responses properly aligned with the flow direction have a high correlation. The primary contribution to the decorrelation between the signals is the spatial shift due to the movement of the scatterers. Another minor contribution is due to new scatterers entering the directional signal at one end and others leaving at the other end. Now consider the case where the directional signals are not aligned along the flow direction. The distribution of the scatterers for the second directional signal is no longer just a shift in position of the previous scatterer distribution. The relative positions between the scatterers have changed, due to the different velocities for the different scatterers. The consequence is that the correlation between the two directional signals is reduced compared to the case of focusing along the flow direction. This change in correlation is the property used for the angle estimation. The method is, thus, to obtain directional signals in a number of directions, and quantify the correlation between signals in each direction. From this procedure a correlation function is constructed showing the normalized correlation as a function of search angle. The maximum of this function indicates the direction with the highest correlation between consecutive signals; hence the flow direction.

The cross-correlation is an obvious choice for the correlation function. However, the cross-correlation peak amplitude is not directly a valid measure. The cross-correlation output values are dependent on the amplitudes of the directional signals, and the amplitudes will differ due to different scatterer distributions at the focal points, and due to the spatial energy distribution of the transmitted ultrasound beam. Instead, the correlation peak should be found from normalized cross-correlation. The normalization factor could be the power of the signal, and this approach is formulated as

$$
\begin{aligned}
R_{12_{n}}(l, \phi) & =\frac{R_{12}(l, \phi)}{P_{12}(\phi)}, \\
R_{12_{\max }}(\phi) & =\max \left(R_{12_{n}}(l, \phi)\right), \\
\hat{\theta}_{k} & =\underset{\phi}{\arg \max }\left\{R_{12_{\max }}(\phi)\right\},
\end{aligned}
$$

where $R_{12_{n}}(l, \phi)$ is the normalized cross-correlation estimate between directional signals $g_{1}\left(x^{\prime}, \phi\right)$ and $g_{2}\left(x^{\prime}, \phi\right)$, and

$$
\begin{aligned}
P_{12}(\phi) & =\sqrt{R_{11}(0, \phi) \cdot R_{22}(0, \phi)} \\
& =\sqrt{\sum_{x^{\prime}} g_{1}\left(x^{\prime}, \phi\right)^{2} \cdot \sum_{x^{\prime}} g_{2}\left(x^{\prime}, \phi\right)^{2}}
\end{aligned}
$$

represents the power of the signals. The term $\phi$ denotes the angle of the directional signal, and $R_{12_{\max }}(\phi)$ is the maximum value of the normalized cross-correlation estimate as a function of beam-angle. The discrete angle estimate $\hat{\theta}_{k}$ is found where $R_{12_{\max }}(\phi)$ has its peak value.

The discrete cross-correlation estimates can yield erroneous peaks when a limited amount of data is used, and if noise is present in the data. This has been investigated in [12]; the influence of noise on the angle estimation is expected to be similar. The cross-correlation estimate can 
be improved by averaging over several directional signals. If averaging is used, $R_{12_{n}}(l, \phi)$ in (11) can be rewritten as

$$
R_{12_{n}}(l, \phi)=\sum_{i} \frac{R_{i, i+1}(l, \phi)}{P_{i, i+1}(\phi)},
$$

where $i$ represents the pulse-echo lines, and the final correlation function from (11) is, thus, formulated as

$$
R_{12_{\max }}(\phi)=\max \left(\sum_{i} \frac{R_{i, i+1}(l, \phi)}{P_{i, i+1}(\phi)}\right) .
$$

The quantization of the cross-correlation is determined from the spatial sampling frequency, when constructing the directional signals. If the spatial sampling interval is sparse, the performance can be improved by interpolation around the peak value of $R_{12_{n}}(l, \phi)$. Fitting a second-order polynomial to the samples around the peak $R_{12}\left(l_{p}, \phi\right)$, where $l_{p}$ denotes the lag at which the peak occurs, will give a more precise maximum value [4], hence a more precise angle estimation.

The correlation between the directional signals can also be described using the correlation coefficient $\rho$. Correlation is the degree to which two or more components are linearly associated, and the statistical term is the covariance

$$
\operatorname{cov}\left(x_{1}, x_{2}\right)=\left\langle\left(x_{1}-\bar{x}_{1}\right)\left(x_{2}-\bar{x}_{2}\right)\right\rangle
$$

between components $x_{1}$ and $x_{2}$, and where \langle\rangle is the expectation operator. The correlation coefficient is simply the covariance normalized with the product of the standard deviations of the two components, and is defined as

$$
\rho=\frac{\operatorname{cov}\left(x_{1}, x_{2}\right)}{\sigma_{x_{1}} \sigma_{x_{2}}}
$$

where $\rho \in[-1,1]$ and $\rho=1$ corresponds to perfect linear relation or full correlation.

The strategy for the angle estimation involves two steps when the correlation coefficient is used for the correlation function. Before calculating the correlation coefficient, the two directional signals, for which the correlation coefficient is needed, are aligned. Otherwise the correlation coefficient will be small, even at the true angle, due to the spatial shift from the movement of the scatterers. The alignment is determined from the discrete lag at the cross-correlation peak $l_{p}$. To increase accuracy, a 2 nd order polynomial is fitted around the peak [4] to find a new interpolated lag value $l_{p}^{\text {int }}$ :

$$
\begin{aligned}
& l_{p}^{\mathrm{int}}= \\
& l_{p}-\frac{R_{12_{n}}\left(l_{p}+1\right)-R_{12_{n}}\left(l_{p}-1\right)}{2\left(R_{12_{n}}\left(l_{p}+1\right)-2 R_{12_{n}}\left(l_{p}\right)+R_{12_{n}}\left(l_{p}-1\right)\right)} .
\end{aligned}
$$

Due to the new continuous lag, the alignment operation involves a first-order linear interpolation or a higher-order interpolation scheme between the samples in one of the signals $g_{1}$ or $g_{2}$. The correlation function using the correlation coefficient is, thus, defined as

$$
\rho(\phi)=\sum_{i} \frac{\operatorname{cov}\left(g_{i}\left(x^{\prime}, \phi\right), g_{i+1}\left(x^{\prime}+l_{p}^{\text {int }}, \phi\right)\right)}{\sigma_{g_{i}\left(x^{\prime}, \phi\right)} \sigma_{g_{i+1}\left(x^{\prime}+l_{p}^{\text {int }}, \phi\right)}},
$$

and the final angle estimation using this function is

$$
\hat{\theta}_{k}=\underset{\phi}{\arg \max }\{\rho(\phi)\},
$$

where the estimate has been improved by averaging over several estimates of $\rho$ using several pulse-echo lines $i$.

If the expectation operator is approximated by the temporal average, and assuming $\bar{x}_{1}=\bar{x}_{2}=0$, then the correlation coefficient of (16) can be written as

$$
\rho=\frac{\sum x_{1} x_{2}}{\sqrt{\sum x_{1}^{2}} \sqrt{\sum x_{2}^{2}}},
$$

and the correlation function can be calculated by rewriting (18) into

$$
\rho(\phi)=\sum_{i} \frac{\sum_{x^{\prime}} g_{i}\left(x^{\prime}, \phi\right) \cdot g_{i+1}\left(x^{\prime}+l_{p}^{\mathrm{int}}, \phi\right)}{\sqrt{\sum_{x^{\prime}} g_{i}\left(x^{\prime}, \phi\right)^{2} \cdot \sum_{x^{\prime}} g_{i+1}\left(x^{\prime}+l_{p}^{\mathrm{int}}, \phi\right)^{2}}},
$$

or in terms of the cross-correlation,

$$
\rho(\phi)=\sum_{i} \frac{R_{i, i+1}^{*}(0, \phi)}{P_{i, i+1}^{*}(\phi)},
$$

where ${ }^{*}$ denotes operation on the aligned signals.

The two approaches to calculating the correlation function in (14) and (21) (or (22)), respectively, are closely related but there are some differences. The quantifier in (21) benefits from the directional signals being accurately aligned before multiplication and summing contrary to the quantifier in (14). Here the summing of cross-correlation functions will suffer from a sparse spatial sampling and they will not sum fully coherently. The quantifier in (21) though requires additional computation due to the interpolation involved in the alignment. The influence of the choice of quantifier on the angle estimation performance is discussed in Section IV.

The directional lines are placed with an angular distance $\Delta \phi$, and the estimates are, thus, initially restricted to be discrete estimates $\hat{\theta}_{k}$ with the same resolution. Continuous estimates $\hat{\theta}$ can be obtained by employing interpolation similar to (17) around the peak of the correlation function to find a continuous index for the peak and then using linear interpolation between the neighboring discrete angles. This will reduce the number of directions to beamform signals in. Prior knowledge of the actual flow angle can also be used in confining the angle search range, with the purpose of limiting the computational task. Iterative schemes could be used, where a temporary estimate based on interpolation between results from a few search 
angles is used. A new search, in a new confined search range around this temporary estimate, can now be made with an increased search resolution. This process continues until the desired precision is obtained or until the limit on the accuracy of the method has been reached.

In the angle and velocity estimator, the correlation is traditionally calculated based on consecutive directional signals. Considering the velocity estimator, for low velocities or high pulse repetition frequencies, the shift between signals is low, yielding a value of $\xi_{x_{\max }^{\prime}}$ from (8) close to zero. This will make the relative estimation variance high since noise will dominate the estimate. It can be an advantage to correlate signals, where the time between acquisitions is higher. The directional signals used for the cross-correlations are then selected with a time interval $T_{c c}=k_{\text {tprf }} \cdot T_{\text {prf }}$ between the correlated signals. $T_{c c}$ is referred to as the correlation time and the proportionality $k_{\text {tprf }}$ is referred to as the correlation time factor. The correlation time factor is, thus, a representation of how many directional signals are bypassed before the correlation is performed. All indices equal to $i+1$ in all previous equations for both velocity and angle estimation must then be substituted by $i+k_{\text {tprf }}$, and, e.g., (5) is then written as

$$
R_{i}\left(\xi_{x^{\prime}}\right)=\int_{X} g_{i}\left(x^{\prime}\right) g_{i+k_{\mathrm{tprf}}}\left(x^{\prime}+\xi_{x^{\prime}}\right) d x^{\prime}, \quad i=1 \ldots N_{x c}
$$

where $g_{i}$ is the $i$ 'th directional signal. Considering the angle estimator, the same dependency on the choice of $k_{\text {tprf }}$ applies, and it appears to be an important parameter which affects performance radically, and the optimal value varies with the flow angle. This will be discussed in more detail in Section IV. The results presented here are all produced with a fixed value for velocity estimation and a fixed value for angle estimation, if nothing else is noticed.

The consequence of using a wrong beam-to-flow angle is different in conventional velocity estimation [1], [2] than with the directional beamforming approach proposed in this paper. With the conventional velocity estimation approach, the estimate available is the velocity projected along the ultrasound beam direction, and this estimate must, thus, be compensated with the beam-to-flow angle to obtain the correct velocity amplitude along the flow. If the angle estimate is not correct, a bias will occur in the velocity estimate. The bias will increase for the same absolute angle estimation error as the true flow angle increases toward $90^{\circ}$. This can be seen from the expression of the relative change in velocity estimate

$$
\frac{\Delta v}{v}=\frac{\cos (\phi)}{\cos (\hat{\phi})}-1
$$

With the velocity estimation approach suggested in this paper, the beam-to-flow angle estimate is used for beamforming the focus lines, and the consequence on the velocity bias is related only to the cosine relation between the true angle and the estimated angle. In Fig. 3, the relative change in velocity estimate is shown as a function of beam-to-flow estimation error for both velocity estimation approaches, with angle errors of $\pm 10^{\circ}$ assuming velocity projection. For the conventional approach, the relative change is shown for $\theta=45^{\circ}$ and $\theta=60^{\circ}$.

\section{RESUlts}

In Section IV-A and Section IV-B, the results from simulations and measurements are presented. The emphasis is placed on the velocity vector angle estimation algorithm. All results are obtained at tube angles of $\theta=\left\{45^{\circ}, 60^{\circ}\right.$, $\left.75^{\circ}, 90^{\circ}\right\}$ and the angle estimation is performed at the vessel center. Furthermore, a study of performance as a function of the parameters $N_{x c}$ and $k_{\text {tprf }}$ is presented. Some of the parameters and processing involved in calculating and presenting the estimation results are described below.

A number $N_{x c}$ of directional lines are cross-correlated for each velocity profile estimate and angle estimate. Echo cancellation is performed by subtracting the mean value of the directional signals used for the estimation.

Specifically for the velocity profile estimation, discrimination between flow and stationary signal is necessary to remove estimates that are based primarily on noise. This rejection scheme is based on the ratio $E_{r}\left(z_{k}\right)$ between the energy of the directional signals after echo cancellation $E_{\mathrm{ec}}$ and before echo cancellation $E$ :

$$
E_{r}\left(z_{k}\right)=\frac{E_{\mathrm{ec}}}{E}=\frac{\sum_{i=1}^{N_{\mathrm{pe}}} \sum_{j=1}^{N_{g}} g_{i_{\mathrm{ec}}}^{2}\left(j, z_{k}\right)}{\sum_{i=1}^{N_{\mathrm{pe}}} \sum_{j=1}^{N_{g}} g_{i}^{2}\left(j, z_{k}\right)},
$$

where $N_{g}$ is the number of samples in the directional signals, $N_{\text {pe }}$ is the number of pulse-echo lines used for the estimate, and $z_{k}$ is the discrete index proportional to the depth. A purely stationary target will yield noise only after echo cancelling, and the ratio will, thus, be low; a proper threshold value is therefore selected for the discrimination.

The results presented are quantified by calculating bias, standard deviations, and probabilities. The velocity profile for a parabolic laminar flow can be described as [2]

$$
v(r)=v_{0}\left(1-\left(\frac{r}{R}\right)^{2}\right)
$$

where $R$ is the tube radius, $v_{0}$ is the maximum velocity attained at the center of the tube, and $r$ is the radial distance from the center of the tube. This is the profile used in the simulated flow, and it is used as reference when calculating the velocity estimation performance in the experimental measurements. The bias and standard deviation are calculated over the profile as a function of depth:

$$
\begin{aligned}
B_{\text {est }}\left(z_{k}\right) & =\overline{\hat{v}}\left(z_{k}\right)-v\left(z_{k}\right), \\
\sigma_{\text {est }}\left(z_{k}\right) & =\sqrt{\frac{1}{N_{\text {est }}-1} \sum_{i=1}^{N_{\text {est }}}\left(\hat{v}_{i}\left(z_{k}\right)-\overline{\hat{v}}\left(z_{k}\right)\right)^{2}},
\end{aligned}
$$



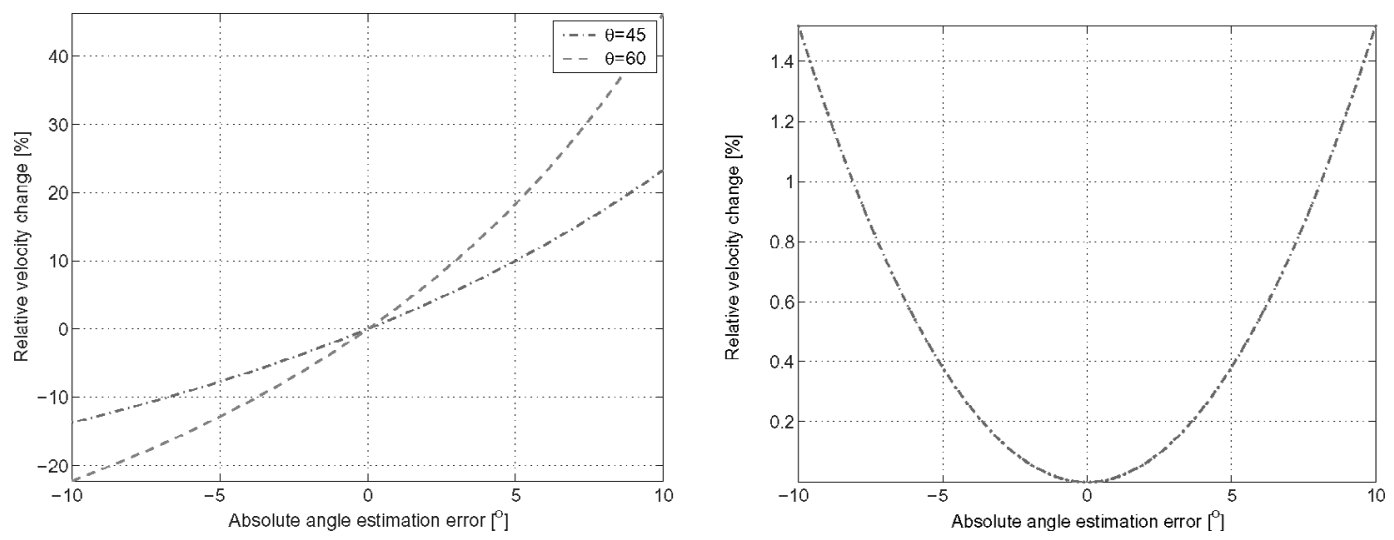

Fig. 3. Velocity estimation error due to a wrong beam-to-flow angle estimate: conventional velocity estimation approach for two different flow directions (left); directional velocity estimation approach (right).

where $z_{k}$ is the discrete depth where the directional signals are obtained, $N_{\text {est }}$ is the total number of velocity profile estimates, $v\left(z_{k}\right)$ is the true profile, and $\overline{\hat{v}}\left(z_{k}\right)$ is the mean profile estimate. From the bias profile $B_{\text {est }}\left(z_{k}\right)$ and standard deviation profile $\sigma_{\text {est }}\left(z_{k}\right)$, the relative mean bias and the relative mean standard deviation are calculated for the whole profile as

$$
\begin{aligned}
B_{\text {rel }} & =\frac{1}{v_{0} \cdot N_{\text {lines }}} \sum_{z_{k}} B_{\text {est }}\left(z_{k}\right), \\
\sigma_{\text {rel }} & =\frac{1}{v_{0}} \sqrt{\frac{1}{N_{\text {lines }}} \sum_{z_{k}} \sigma_{\text {est }}^{2}\left(z_{k}\right)},
\end{aligned}
$$

where $N_{\text {lines }}$ is the number of directional lines, or the number of velocity estimates in one profile.

Since the kernel of the angle estimation algorithm is the cross-correlation, there is a risk of having estimates that are based on erroneous peaks. These estimates can take values much different from the true angle. If no attention is made to these outlier estimates, the bias and the standard deviation of all estimates will be highly influenced by these and will not represent the performance properly. When angle estimates in some form of blood flow imaging are visualized, a few false estimates in a group of almost correct estimates can easily be handled by the human eye. As a consequence of these aspects, the angle estimates are divided into two groups: one group with estimates that are within a specified acceptable deviation $\theta_{\text {dev }}$ from the true angle, and one group with the complementary estimates, which are considered outliers. A probability of having a continuous estimate $\hat{\theta}$ within $\theta_{\text {dev }}$ from the true angle is calculated as

$$
P_{\mathrm{ok}}=P\left(\theta-\theta_{\mathrm{dev}} \leq \hat{\theta} \leq \theta+\theta_{\mathrm{dev}}\right)
$$

where $\theta_{\text {dev }}=15^{\circ}$ by choice. All estimates outside this range are considered outliers. The bias $B_{\text {est }}$ and standard
TABLE I

Default Parameters for the Velocity Profile and Angle Estimation Algorithm.

\begin{tabular}{lc}
\hline Spatial sampling interval for directional signals & $\lambda / 10$ \\
Spatial extent of directional signals & $-10 \lambda: 10 \lambda$ \\
(correlation interval) & \\
$\begin{array}{l}\text { Number of correlations for each estimate } \\
\theta=\left\{45^{\circ}, 60^{\circ}, 75^{\circ}, 90^{\circ}\right\}\end{array}$ & $\{10,10,10,20\}$ \\
Angular distance between directional lines & $5^{\circ}$ \\
for angle estimation & 8 \\
Correlation time factor $k_{\text {tprf }}$ for angle & \\
estimation & Center of vessel \\
Position of angle estimate & $1 \mathrm{~m} / \mathrm{s}$ \\
Velocity search range & 2 \\
Correlation time factor $k_{\text {tprf }}$ for velocity & \\
estimation &
\end{tabular}

deviation $\sigma_{\text {est }}$ are calculated based on that subset of estimates only, which are within $\theta_{\text {dev }}$ from the true angle:

$$
\begin{aligned}
B_{\text {est }} & =\overline{\hat{\theta}}-\theta, \\
\sigma_{\text {est }} & =\sqrt{\frac{1}{N_{\text {sub }}-1} \sum_{i=1}^{N_{\text {sub }}}\left(\hat{\theta}_{i}-\overline{\hat{\theta}}\right)^{2}},
\end{aligned}
$$

where $N_{\text {sub }}$ is the number of estimates in the subset, and $\overline{\hat{\theta}}$ is the mean estimate within the subset.

The default parameters for velocity and angle estimation are found in Table I.

In Section III two different ways of quantifying the correlation between the directional signals were described. One was to use the normalized amplitude from the crosscorrelation, and the other was to use the correlation coefficient. All angle estimation results presented here are produced with the correlation coefficient for the correlation function if nothing else is noticed. A complete comparison study of the performance with the two different quantifiers has not been made, but examples of the differences will be given in Section IV-B,3. 
TABLE II

Default Parameters Used in All Simulations.

\begin{tabular}{lc}
\hline Number of transmit elements & 128 \\
Number of receive elements & 128 \\
Transducer center frequency & $7 \mathrm{MHz}$ \\
Pitch of transducer element & $0.208 \mathrm{~mm}$ \\
Height of transducer element & $4.5 \mathrm{~mm}$ \\
Kerf & $0.035 \mathrm{~mm}$ \\
Assumed speed of sound & $1540 \mathrm{~m} / \mathrm{s}$ \\
Transmit apodization & Hanning \\
Receive apodization & Rectangular (none) \\
Excitation pulse & $1-$ period sinusoid \\
Pulse repetition frequency & $10 \mathrm{kHz}$ \\
RF sampling frequency & $100 \mathrm{MHz}$ \\
Radius of vessel & $10 \mathrm{~mm}$ \\
Distance to vessel center & $40 \mathrm{~mm}$ \\
Transmit focus & $80 \mathrm{~mm}$ \\
Peak velocity in flow, $v_{0}$ & $0.3 \mathrm{~m} / \mathrm{s}$ \\
\hline
\end{tabular}

TABLE III

Velocity Profile Estimates from Field II Simulations.*

\begin{tabular}{cccc}
\hline$\theta$ & $\begin{array}{c}\text { Relative mean } \\
\text { bias [\%] }\end{array}$ & $\begin{array}{c}\text { Relative mean } \\
\text { standard deviation [\%] }\end{array}$ & $\begin{array}{c}\text { Estimates } \\
\text { in profile }\end{array}$ \\
\hline $45^{\circ}$ & 0.4 & 0.7 & 29 \\
$60^{\circ}$ & 1.2 & 1.0 & 29 \\
$75^{\circ}$ & 3.5 & 1.8 & 27 \\
$90^{\circ}$ & -3.6 & 7.7 & 27 \\
\hline
\end{tabular}

${ }^{*}$ Results are calculated from estimates inside the vessel only.

\section{A. Simulation Results Using Field II}

The main purpose of the simulations is to verify the functionality of the algorithms, and to evaluate how good the performance can get under favorable conditions. In Field II the conditions are ideal, and the simulation results are obtained from acquired data free from noise. The velocity and angle estimates are estimated from the same simulated data, with transmit focus at twice the depth of the vessel center. The simulation results are presented similar to the measurement results, using the same parameters with a few exceptions. The default parameter values for the simulations are found in Table II.

1. Velocity Profile Estimation on Simulated Data: The results are based on 1000 pulse-echo signals which lead to 165 and 89 profile estimates for the beam-to-flow angles of $45^{\circ}$ to $75^{\circ}$ and $90^{\circ}$, respectively. The profiles are calculated using the flow/stationary rejection scheme, and the beamto-flow angle is assumed known.

The mean velocity profile and an indication of the deviation from this mean profile are illustrated in Fig. 4 for all four flow angles. The performance is calculated from the estimates inside the vessel only and can be found in Table III. Both the bias and the standard deviation increase with flow angle, and the standard deviation for transverse flow is noticeable larger than for the other angles.
TABLE IV

Result of Angle Estimation from Simulation in Field II.

\begin{tabular}{cccc}
\hline Flow angle, & Probability, & Bias, & $\begin{array}{c}\text { Standard } \\
\text { deviation } \\
\theta\end{array}$ \\
$P_{\text {ok }}$ & \multicolumn{1}{c}{$B_{\text {est }}$} & $\sigma_{\text {est }}$ \\
\hline $45^{\circ}$ & $100 \%$ & $-0.6^{\circ}$ & $2.9^{\circ}$ \\
$60^{\circ}$ & $100 \%$ & $-1.5^{\circ}$ & $2.2^{\circ}$ \\
$75^{\circ}$ & $97 \%$ & $-4.9^{\circ}$ & $2.8^{\circ}$ \\
$90^{\circ}$ & $89 \%$ & $0.3^{\circ}$ & $0.7^{\circ}$ \\
\hline
\end{tabular}

TABLE V

Result of Angle Estimation from Simulations with Optimal Values of the Correlation Time $k_{\text {TPRF }}$.

\begin{tabular}{ccccc}
\hline Flow angle, & & $\begin{array}{c}\text { Probability, } \\
P_{\text {ok }}\end{array}$ & $\begin{array}{c}\text { Bias, } \\
B_{\text {est }}\end{array}$ & $\begin{array}{c}\text { Standard } \\
\text { deviation, } \\
\sigma_{\text {est }}\end{array}$ \\
\hline $45^{\circ}$ & 18 & $100 \%$ & $-0.4^{\circ}$ & $1.9^{\circ}$ \\
$60^{\circ}$ & 18 & $100 \%$ & $-0.9^{\circ}$ & $1.7^{\circ}$ \\
$75^{\circ}$ & 18 & $100 \%$ & $-1.0^{\circ}$ & $0.4^{\circ}$ \\
$90^{\circ}$ & 6 & $100 \%$ & $0.4^{\circ}$ & $0.9^{\circ}$ \\
\hline
\end{tabular}

2. Angle Estimation on Simulated Data: The simulation results in this section are based on the default simulation parameters from Table II and the default angle estimation parameters from Table I.

The results are based on 1000 pulse-echo acquisitions which lead to 110 and 70 angle estimates for the beamto-flow angles $45^{\circ}, 60^{\circ}, 75^{\circ}$, and $90^{\circ}$, respectively. The performance is shown in Table IV.

The performance decreases as the flow angle increases from $\theta=45^{\circ}$ to $\theta=90^{\circ}$. At $\theta=90^{\circ}$ there are dominant secondary peaks at the correlation function which divides the estimates into a few groups, giving relatively many outliers at specific angles. However, those estimates that are within the acceptable deviation have a low bias and standard deviation. It should be emphasized that this performance dependency on flow angle is different with another choice of correlation time factor $k_{\text {tprf }}$. Since the choice of $k_{\text {tprf }}$ has a strong influence on performance, a study of this influence is performed and described in Section IV-B,3, based on measured data. A similar study is performed on simulated data with values of $k_{\mathrm{tprf}}$ from 2 to 18 to find those values which optimize the performance for flow angles of $\theta=\left\{45^{\circ}, 60^{\circ}, 75^{\circ}, 90^{\circ}\right\}$. The results of the angle estimation with these optimal values are shown in Table $\mathrm{V}$ with the only purpose of illustrating the absolute potential of the method under favorable conditions.

\section{B. Experimental Measurements}

This section describes the measurement setup and presents the angle estimation results from the experiments.

1. Measurement Setup: The experimental ultrasound scanner RASMUS [15] and a 7-MHz linear array trans- 

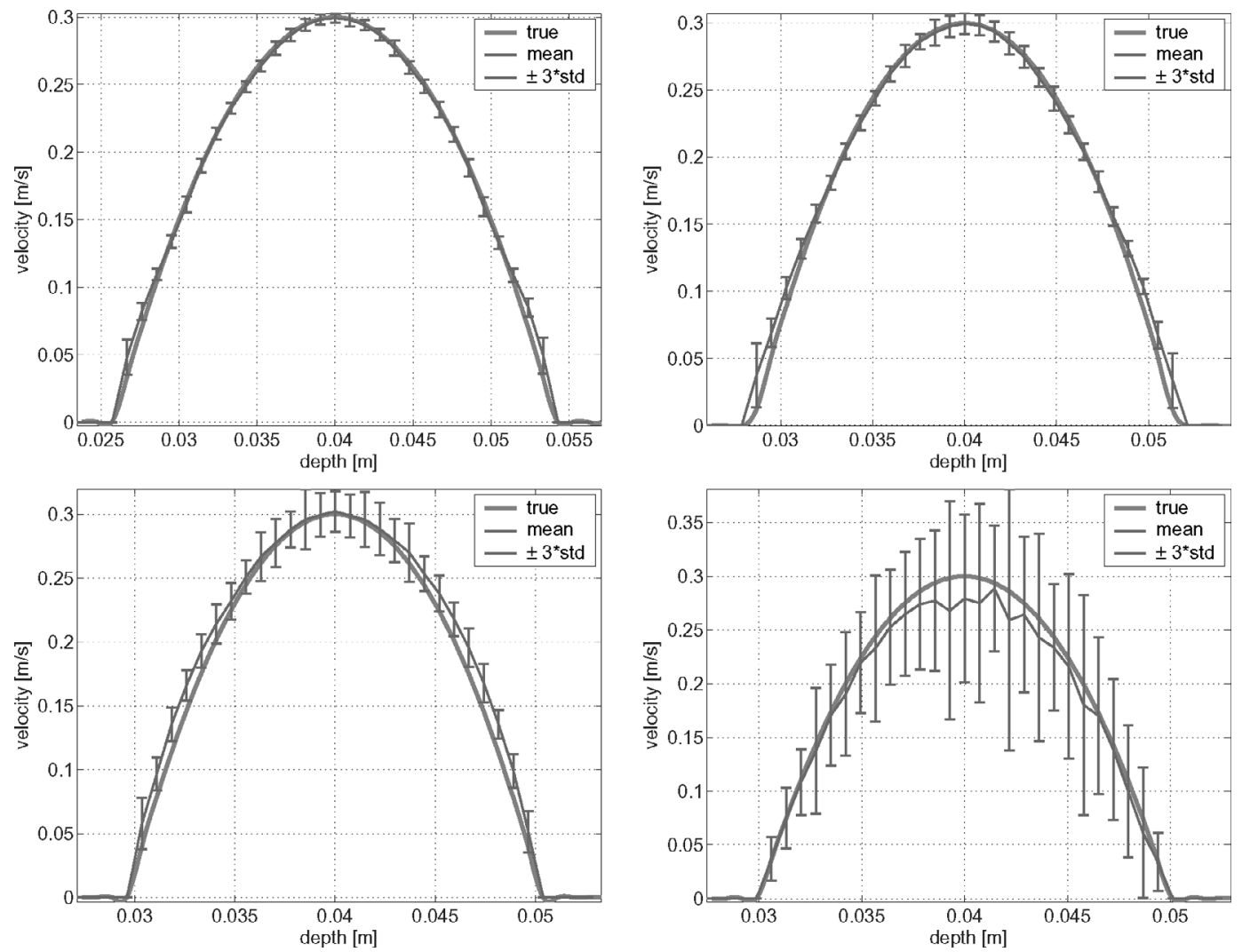

Fig. 4. Velocity profile estimation from simulations in Field II: mean profile and standard deviation of profile estimates. Flow/stationary rejection scheme is used. $\theta=45^{\circ}$ (upper left), $\theta=60^{\circ}$ (upper right), $\theta=75^{\circ}$ (lower left), $\theta=90^{\circ}$ (lower right).

ducer, type 8804, from B-K Medical (DK-2730 Herlev, Denmark), are used for all measurements. The system acquires RF data from the individual transducer channels and these data are transferred to a PC, where they are saved and processed off-line. The RASMUS system contains 128 transmitting channels and 64 receiving channels with a $40-\mathrm{MHz}$ sampling frequency, and has 12-bit resolution for each individual channel. With the built-in 2-to-1 multiplexer, the system can cover 128 individual receive channels in two receptions.

All measurements are made using a circulating flow rig with a blood-mimicking fluid. A pump is connected to a steel tube of approximately $1 \mathrm{~m}$, which enters a water tank; there the steel tube enters another tube made of heat shrink, which mimics a blood vessel. This tube is connected to a similar steel tube before it exits the water tank. Inside the water tank a fixture for the transducer is inserted. The angle of the transducer and the distance to the tube can be adjusted. The distance from the center element of the transducer to the tube is the same regardless of the angle. The measurements are obtained at the angles $45^{\circ}, 60^{\circ}, 75^{\circ}$, and $90^{\circ}$, where $90^{\circ}$ corresponds to a transverse flow measurement.

The measurement setup consists of a blood-mimicking fluid and a PC-controlled pump (CompuFlow1000, Shelley Medical Imaging Technologies, London, Ontario, Canada) [16]. In CompuFlow1000 a piston is moved and maintains a constant flow for a period of time. The reference velocity is based solely on volume flow input to the control PC.
TABLE VI

Default Measurement Parameters Used for All Measurements.

\begin{tabular}{lc}
\hline Number of transmit elements & 128 \\
Number of receive elements & $64 / 128$ (combined) \\
Pitch of transducer element & $0.208 \mathrm{~mm}$ \\
Height of transducer element & $4.5 \mathrm{~mm}$ \\
Kerf & $0.035 \mathrm{~mm}$ \\
Transmit apodization & Hanning \\
Receive apodization & Rectangular (none) \\
Excitation pulse & 2 -cycle sinusoid \\
Pulse repetition frequency & $10 \mathrm{kHz} / 5 \mathrm{kHz}($ combined) \\
RF sampling frequency & $40 \mathrm{MHz}$ \\
Transducer center frequency & $7 \mathrm{MHz}$ \\
Assumed speed of sound & $1480 \mathrm{~m} / \mathrm{s}$ \\
Radius of vessel & $6.4 \mathrm{~mm}$ \\
Distance to vessel center & $37 \mathrm{~mm}$ \\
Peak velocity in flow & $\approx 0.3 \mathrm{~m} / \mathrm{s}$ \\
\hline
\end{tabular}

Inaccuracy in the control system and the loss throughout the flow rig system will contribute to a bias in the estimate. For all measurements, a number of parameters has been fixed and these are found in Table VI.

The system is capable of receiving data from 64 channels simultaneously. By use of the built-in 2-to-1 multiplexer, the system can receive data from 128 channels in 2 emissions. For the odd-numbered emissions the first 64 channels of the transducer are used in receive, and for the 
TABLE VII

Channels Used in Receive Mode.

\begin{tabular}{lcccccccc}
\hline Emission number & 1 & 2 & 3 & 4 & 5 & 6 & $\ldots$ & $N_{\text {shots }}$ \\
Receive channels & $1: 64$ & $65: 128$ & $1: 64$ & $65: 128$ & $1: 64$ & $65: 128$ & $\ldots$ & \\
\hline
\end{tabular}

TABLE VIII

Exception from the Default Parameters of Table I Used for Angle Estimation on Measured Data.

Number of correlations for each estimate $\theta=\left\{45^{\circ}, 60^{\circ}, 75^{\circ}, 90^{\circ}\right\}$

$\{20,20,20,20\}$

TABLE IX

Result of Angle Estimation from Measurements Using the Correlation Coefficient for the Correlation Function.

\begin{tabular}{cccc}
\hline $\begin{array}{c}\text { Flow angle, } \\
\theta\end{array}$ & $\begin{array}{c}\text { Probability, } \\
P_{\text {ok }}\end{array}$ & $\begin{array}{c}\text { Bias, } \\
B_{\text {est }}\end{array}$ & $\begin{array}{c}\text { Standard } \\
\text { deviation, } \\
\sigma_{\text {est }}\end{array}$ \\
\hline $45^{\circ}$ & $62 \%$ & $-1.5^{\circ}$ & $8.1^{\circ}$ \\
$60^{\circ}$ & $95 \%$ & $-2.8^{\circ}$ & $4.3^{\circ}$ \\
$75^{\circ}$ & $98 \%$ & $-2.0^{\circ}$ & $2.0^{\circ}$ \\
$90^{\circ}$ & $68 \%$ & $0.4^{\circ}$ & $1.0^{\circ}$ \\
\hline
\end{tabular}

even-numbered emissions the last 64 channels are used, as shown in Table VII.

The first 128-channel combined data matrix $C_{1}$ are available after the 2 nd emission by simply putting together the data from the emission $1, d_{1}$ and data from emission $2, d_{2}$. The second 128-channel data matrix $C_{2}$ is available after the $3 \mathrm{rd}$ emission by putting together $d_{3}$ and $d_{2}$. The list of 128-channel combined data $\mathbf{C}$ can thus be written as

$$
\begin{aligned}
\mathbf{C}= & \left\{C_{1}, C_{2}, C_{3}, \ldots, C_{N_{\text {shots }}-1}\right\} \\
= & \left\{\left[d_{1}, d_{2}\right],\left[d_{3}, d_{2}\right],\left[d_{3}, d_{4}\right],\left[d_{5}, d_{4}\right],\left[d_{5}, d_{6}\right], \ldots,\right. \\
& {\left.\left[d_{N_{\text {shots }}-1}, d_{N_{\text {shots }}}\right]\right\} . }
\end{aligned}
$$

Notice that the even-numbered combined data are obtained differently than the odd-numbered data. The crosscorrelation of combined and beamformed signals should, thus, be done only between even-numbered or oddnumbered $C$-data.

2. Angle Estimation on Measured Data: The results in this section are based on the default parameters from Table I and the exception in Table VIII. Three thousand pulse-echo acquisitions are processed, which leads to 213 angle estimates.

The estimation performance is found in Fig. 5 for the four different flow angles. They show the probability $P_{\mathrm{ok}}$ and the distribution of those continuous estimates that are not classified as outliers. A summary of the quantized performance can be found in Table IX. The performance at $\theta=45^{\circ}$ is radically worse than at the other angles. Velocity profile estimates have been calculated based on the same acquisition data, and here there was a similar decrease in performance. One thing to notice about the measurement setup is that there is a relatively large difference in physical position of the transducer for the different tube angles. The distance from the center element to the tube is the same regardless of the angle, but for $\theta=45^{\circ}$ the transducer element in one end is very close to the tube, which could have a negative effect in form of re-reflections. Re-reflections inside the water tank can also have some influence on the results, and this will also vary with the angle of the transducer. Another thing to notice is that the simulation did not show any decrease in performance for $\theta=45^{\circ}$ compared to the other angles.

The correlation function is the basis of the angle estimation. The correlation functions for all estimates are superimposed for a given flow angle in Fig. 6. This figure shows how certain or reliable the estimates are. The shape of the correlation function and specifically the amplitude distribution and the position of the peaks are interesting. From this figure it is also evident that the performance for $\theta=45^{\circ}$ is worse than at the other angles. At the other angles the peak around the true angle is much more distinct and the coefficient attenuates faster as $\phi$ moves away from the true angle. For $\theta=90^{\circ}$ there are two dominant peaks adjacent to the peak at $\theta=90^{\circ}$. This shape is consistent with the lower-left histogram in Fig. 5 where the estimates are divided into one dominant group around $\theta=90^{\circ}$ and two minor groups around $\theta=65^{\circ}$ and $\theta=115^{\circ}$, which was also the case with the simulated data.

3. Angle Estimation Parameter Study: In this section, studies of performance as a function of $k_{\mathrm{tprf}}$ and $N_{x c}$ are carried out. The study of $k_{\text {tprf }}$ will be done with $N_{x c}=20$ and the study of $N_{x c}$ with $k_{\text {tprf }}=8$ for all flow angles.

The angle estimation results in Section IV-B,2 were all produced with a fixed value of $k_{\mathrm{tprf}}$, and the study in this section will show how different values affect performance. In Fig. 7, the study of $k_{\text {tprf }}$ for all flow angles is shown. In each figure, $P_{\mathrm{ok}}$ is plotted as a function of $k_{\mathrm{tprf}}$, and so are the bias $b_{\text {est }}$ and the standard deviation $\sigma_{\text {est }}$. It is evident from these figures that the choice of $k_{\text {tprf }}$ has a strong influence on performance and that the optimal value varies with the flow angle. The optimal value is in some degree a subjective choice of whether to optimize $P_{\text {ok }}, b_{\text {est }}$, or $\sigma_{\text {est }}$, but desirable values for the given data set for $\theta=\left\{45^{\circ}\right.$, $\left.60^{\circ}, 75^{\circ}, 90^{\circ}\right\}$ are $k_{\text {tprf }}=\{18,18,20,4\}$. For the angles $\theta=\left\{45^{\circ}, 60^{\circ}, 75^{\circ}\right\}$ the estimation benefits from having a high value quite contrary to the case for $\theta=90^{\circ}$, where a low value is preferable. This is likely due to the low lateral oscillation of the field and the fast de-correlation at the true angle, when increasing the correlation time. However, these chosen values can be considered optimal only for the 

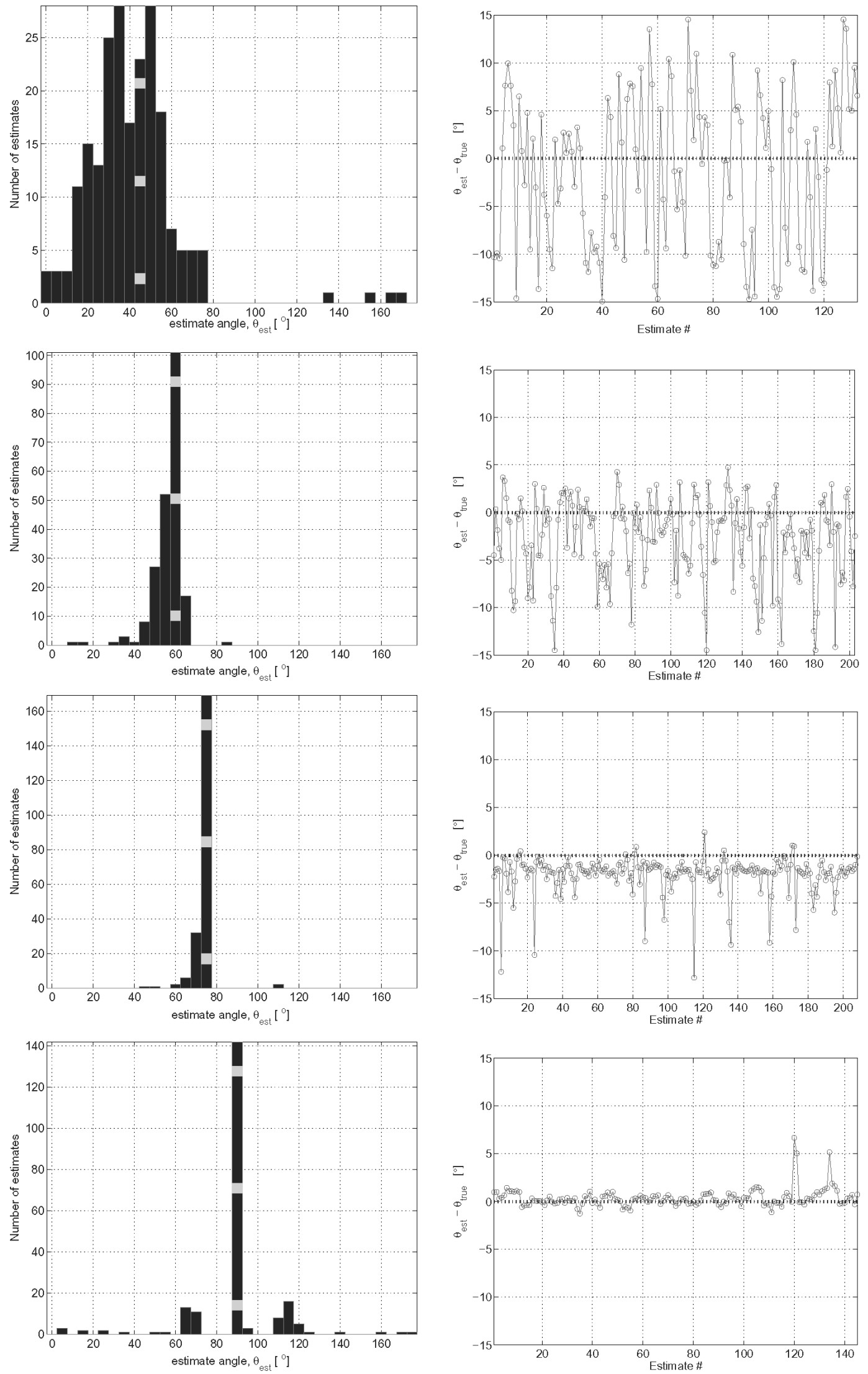

Fig. 5. Angle estimation from measurements for $\theta=45^{\circ}, 60^{\circ}, 75^{\circ}$, and $90^{\circ}$ : a histogram of the discrete estimates (left); deviation between accepted continuous estimates and the true angle (right). 

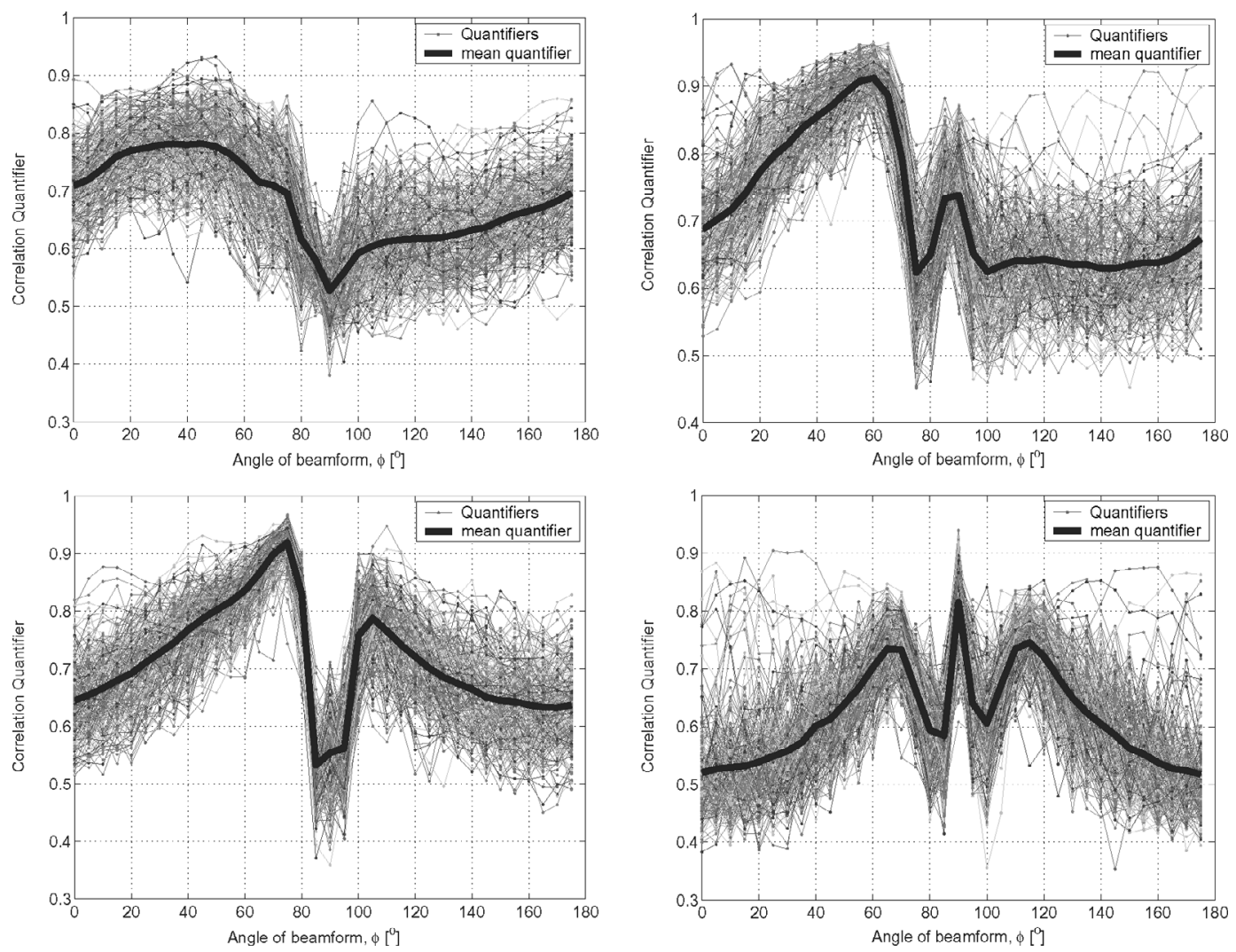

Fig. 6. Angle Estimation: coefficient functions for all estimates are superimposed for $\theta=45^{\circ}$ (upper left), $\theta=60^{\circ}$ (upper right), $\theta=75^{\circ}$ (lower left), and $\theta=90^{\circ}$ (lower right).
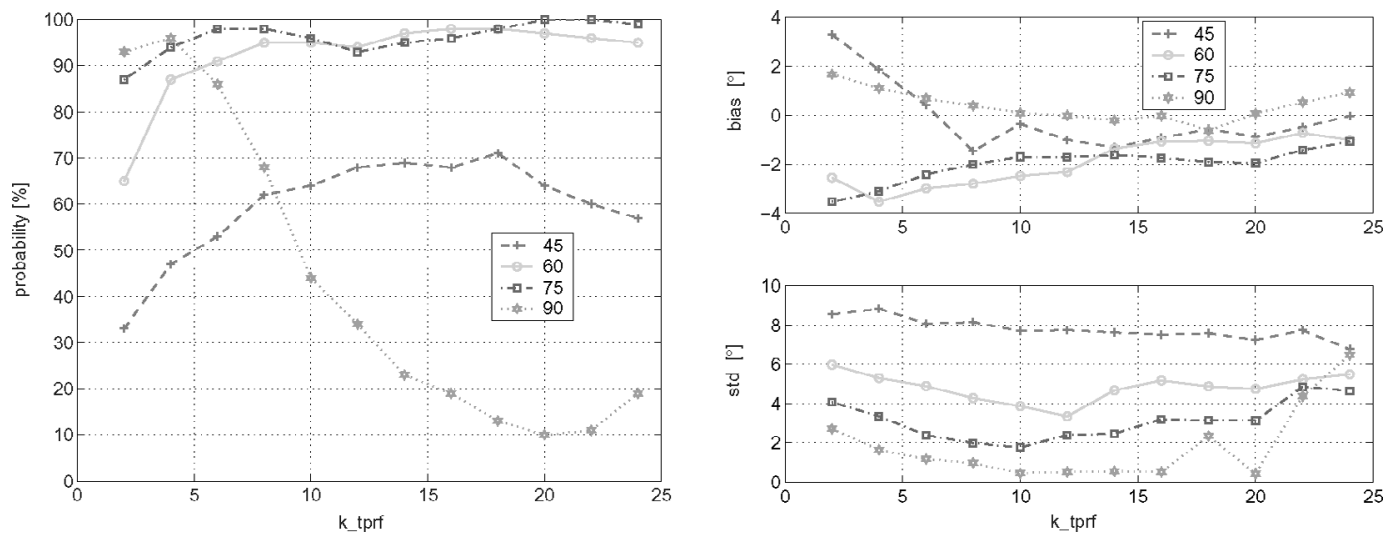

Fig. 7. Performance as a function of $k_{\mathrm{tprf}}$ for $\theta=45^{\circ}, 60^{\circ}, 75^{\circ}$, and $90^{\circ}$ : probability of having an estimate within $\pm 15^{\circ}$ from the true angle (left); bias $B_{\text {est }}$ and standard deviation $\sigma_{\text {est }}$ of that subset of continuous estimates that are within $\pm 15^{\circ}$ from the true angle (right).

data used in the parameter study. Using another set of data with a different flow velocity or different signal-to-noise ratio, the set of optimal values would likely be different. The angle estimation performance with $k_{\text {tprf }}=\{18,18,20,4\}$ is shown in Table $\mathrm{X}$ to illustrate the performance under favorable conditions.

The same parameter study on $k_{\text {tprf }}$ has been done using the correlation function based on finding the peak amplitude of the averaged cross-correlation function, as described in Section III. From this study the conclusion on desirable values of $k_{\mathrm{tprf}}$ is unchanged, and a plot show-
TABLE X

Result of Angle Estimation from Measurements with Optimal Values of the Correlation Time $k_{\text {TPRF }}$.

\begin{tabular}{|c|c|c|c|c|}
\hline $\begin{array}{c}\text { Flow angle, } \\
\theta\end{array}$ & $k_{\mathrm{tprf}}$ & $\begin{array}{c}\text { Probability, } \\
P_{\mathrm{ok}}\end{array}$ & $\begin{array}{c}\text { Bias, } \\
B_{\text {est }}\end{array}$ & $\begin{array}{c}\text { Standard } \\
\text { deviation, } \\
\sigma_{\text {est }}\end{array}$ \\
\hline $45^{\circ}$ & 18 & $71 \%$ & $-0.6^{\circ}$ & $7.6^{\circ}$ \\
\hline $60^{\circ}$ & 18 & $98 \%$ & $-1.1^{\circ}$ & $4.9^{\circ}$ \\
\hline $75^{\circ}$ & 20 & $100 \%$ & $-2.0^{\circ}$ & $3.1^{\circ}$ \\
\hline $90^{\circ}$ & 4 & $96 \%$ & $1.1^{\circ}$ & $1.6^{\circ}$ \\
\hline
\end{tabular}



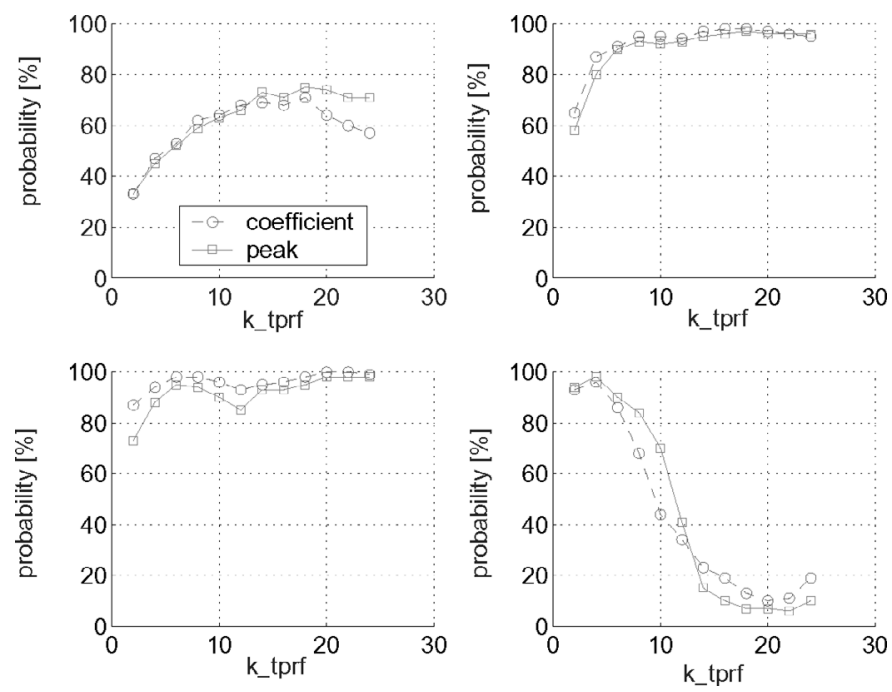

Fig. 8. Performance as a function of $k_{\text {tprf }}$ with two different types of correlation functions for $\theta=45^{\circ}$ (upper left), $\theta=60^{\circ}$ (upper right), $\theta=75^{\circ}$ (lower left), and $\theta=90^{\circ}$ (lower right). Probability of having an estimate within $\pm 15^{\circ}$ from the true angle.

TABLE XI

Result of Angle Estimation from Measurements Using the Cross-Correlation Peak Amplitude for the Correlation FUNCTION.

\begin{tabular}{|c|c|c|c|}
\hline $\begin{array}{c}\text { Flow angle, } \\
\theta\end{array}$ & $\begin{array}{c}\text { Probability, } \\
P_{\mathrm{ok}}\end{array}$ & $\begin{array}{c}\text { Bias, } \\
B_{\text {est }}\end{array}$ & $\begin{array}{c}\text { Standard } \\
\text { deviation, } \\
\sigma_{\text {est }}\end{array}$ \\
\hline $45^{\circ}$ & $59 \%$ & $-1.6^{\circ}$ & $7.7^{\circ}$ \\
\hline $60^{\circ}$ & $93 \%$ & $-3.9^{\circ}$ & $4.7^{\circ}$ \\
\hline $75^{\circ}$ & $94 \%$ & $-2.5^{\circ}$ & $2.3^{\circ}$ \\
\hline $90^{\circ}$ & $84 \%$ & $0.8^{\circ}$ & $1.4^{\circ}$ \\
\hline
\end{tabular}

ing $P_{\mathrm{ok}}$ as a function of $k_{\mathrm{tprf}}$ for both types of correlation functions is shown in Fig. 8.

The result of the angle estimation using the peak correlation for the correlation function and $k_{\text {tprf }}=8$, just as in Table IX and Fig. 5 is shown in Table XI. Only small differences exist when comparing Table IX with Table XI and when observing Fig. 8. The benefits of using the correlation coefficient over the correlation peak amplitude is, thus, questionable when the additional computational task is considered.

If the time in between consecutive directional signals used for the cross-correlation is very small, the two directional signals will be very similar. As stated in Section III, the distribution of scatterers making up the second of the two directional signals is basically the same as the distribution making up the first directional signal. This is due to the extent of the point-spread function. There is a high correlation between the signals also, if they are obtained at an angle different from the flow angle. At some point, the time in between the signals is so large that the physical movement of the scatterers is on the order of the extent of the point-spread function. The signals obtained at angles different from the flow angle will, thus, have a very low correlation because of different scatter distributions. The signals obtained at the flow angle do not have this problem, and the main contribution to the de-correlation is the shift. This is where the optimal value for the correlation time is found, namely, where there is a large difference between the correlation at the flow angle and the other angles, which makes it possible to discriminate. Further time increase will merely result in further de-correlation of the signals at the flow angle, and since the signals at the other angles at this point are almost completely uncorrelated, the discrimination will be more difficult. To illustrate this, the mean correlation function as a function of $k_{\mathrm{tprf}}$ is shown in Fig. 9 for all flow angles. There is a general decrease in correlation with increasing values of $k_{\text {tprf }}$, as expected, and the decrease rate is stronger at angles different from the true angle. For $\theta=90^{\circ}$ the decrease rate at the true angle relative to the other search angles is stronger than for $\theta=45^{\circ}, 60^{\circ}$, and $75^{\circ}$. This is in agreement with the lower desirable value of $k_{\mathrm{tprf}}$ for $\theta=90^{\circ}$ than for $\theta=45^{\circ}, 60^{\circ}$, and $75^{\circ}$ found previously.

If the flow is stationary, the estimate will improve from averaging over a number of correlations $N_{x c}$, and the improvement will increase with a broader averaging. The study of performance as a function of $N_{x c}$ is still interesting, though, since it will show the relation between the performance and the necessary computational task. This study is presented in the same way as the above study of $k_{\text {tprf }}$ and with $k_{\text {tprf }}=8$, just as for the results in Table IX and Fig. 5. The results from all flow angles are shown in Fig. 10. With attention to the results for $\theta=60^{\circ}, 75^{\circ}$, and $90^{\circ}$, it seems possible to decrease the number of outliers radically until $P_{\mathrm{ok}}$ reaches approximately $90 \%$, where further increase of $N_{x c}$ has only little effect.

\section{Conclusion}

When focusing along the velocity direction is used for velocity estimation, the velocity profile estimates from simulations have relative mean standard deviations between $0.7 \%$ and $7.7 \%$ for flow between $45^{\circ}$ and $90^{\circ}$. The simulation study and measurement study both showed that angle estimation by directional beamforming can be estimated with a high precision, potentially yielding velocity estimates with a better accuracy. The angle estimation performance is strongly influenced by the choice of correlation time, and a proper choice varies with flow angle and will vary with flow velocity amplitude, though the latter is not shown in this paper. Flow angles of $\theta=\left\{45^{\circ}, 60^{\circ}\right.$, $\left.75^{\circ}\right\}$ require a high value of the correlation time whereas for $\theta=90^{\circ}$ a low value is required. By using the optimal choice of correlation time for the different flow angles based on a parameter study, the potential of the method has been revealed. With these favorable conditions the simulations produced $100 \%$ valid estimates (no outliers) and a bias and standard deviation below $2^{\circ}$ for all flow angles. Using the measurements, more than $96 \%$ valid estimates 

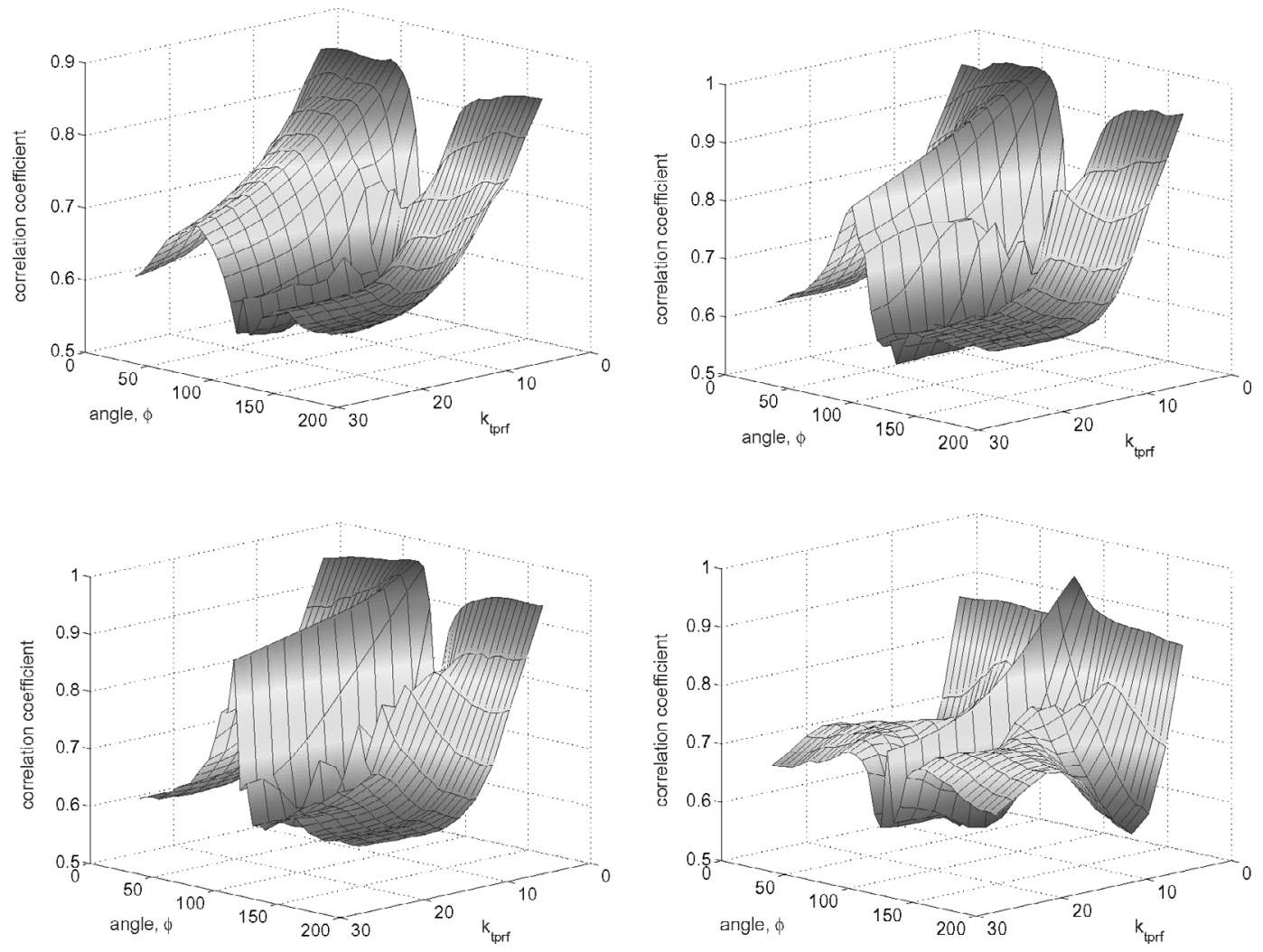

Fig. 9. Mean correlation function as a function of $k_{\mathrm{tprf}}$ for $\theta=45^{\circ}$ (upper left), $\theta=60^{\circ}$ (upper right), $\theta=75^{\circ}$ (lower left), and $\theta=90^{\circ}$ (lower right).
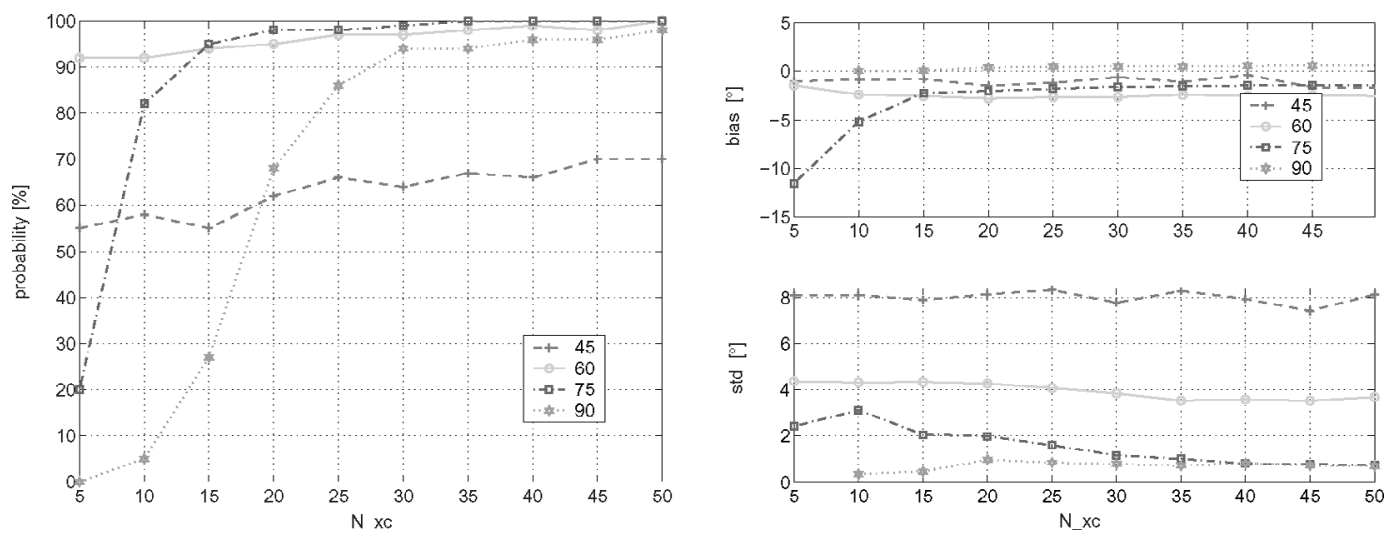

Fig. 10. Performance as a function of $N_{x c}$ for $\theta=45^{\circ}, 60^{\circ}, 75^{\circ}$, and $90^{\circ}$ : probability of having estimates within $\pm 15^{\circ}$ from the true angle (left); bias $B_{\text {est }}$ and standard deviation $\sigma_{\text {est }}$ of that subset of estimates that are within $\pm 15^{\circ}$ from the true angle (right).

were produced for the flow angles $\theta=\left\{60^{\circ}, 75^{\circ}, 90^{\circ}\right\}$ and with a bias below $2^{\circ}$ and a standard deviation below $5^{\circ}$. The techniques used are based on an assumption of a laminar flow. A turbulent flow will evidently complicate matters of angle estimation and can be a topic of future investigations.

\section{REFERENCES}

[1] D. H. Evans, W. N. McDicken, R. Skidmore, and J. P. Woodcock, Dopper Ultrasound, Physics, Instrumentation, and Clinical Applications. New York: John Wiley \& Sons, 1989.
[2] J. A. Jensen, Estimation of Blood Velocities Using Ultrasound: A Signal Processing Approach. New York: Cambridge University Press, 1996.

[3] C. Kasai, K. Namekawa, A. Koyano, and R. Omoto, "Real-time two-dimensional blood flow imaging using an autocorrelation technique," IEEE Trans. Sonics Ultrason., vol. 32, pp. 458-463, 1985.

[4] S. G. Foster, "A pulsed ultrasonic flowmeter employing time domain methods," Ph.D. dissertation, Department of Electrical Engineering, University of Illinois, Urbana, IL, 1985.

[5] O. Bonnefous and P. Pesqué, "Time domain formulation of pulse-Doppler ultrasound and blood velocity estimation by cross correlation," Ultrason. Imag., vol. 8, pp. 73-85, 1986.

[6] M. D. Fox, "Multiple cross-beam ultrasound Doppler velocimetry," IEEE Trans. Sonics Ultrason., vol. SU-25, pp. 281-286, 
1978.

[7] G. E. Trahey, J. W. Allison, and O. T. von Ramm, "Angle independent ultrasonic detection of blood flow," IEEE Trans. Biomed. Eng., vol. BME-34, pp. 965-967, 1987.

[8] J. A. Jensen and P. Munk, "A new method for estimation of velocity vectors," IEEE Trans. Ultrason., Ferroelect., Freq. Contr., vol. 45, pp. 837-851, 1998.

[9] M. E. Anderson, "Multi-dimensional velocity estimation with ultrasound using spatial quadrature," IEEE Trans. Ultrason., Ferroelect., Freq. Contr., vol. 45, pp. 852-861, 1998.

[10] H. F. Routh, T. L. Pusateri, and D. D. Waters, "Preliminary studies into high velocity transverse blood flow measurement," in Proc. IEEE Ultrason. Symp., 1990, pp. 1523-1526.

[11] V. L. Newhouse, D. Censor, T. Vontz, J. A. Cisneros, and B. B. Goldberg, "Ultrasound Doppler probing of flow transverse with respect to beam axis," IEEE Trans. Biomed. Eng., vol. BME-34, pp. $779-788,1987$.

[12] J. A. Jensen, "Directional velocity estimation using focusing along the flow direction: I. Theory and simulation," IEEE Trans. Ultrason., Ferroelect., Freq. Contr., vol. 50, pp. 857-872, 2003.

[13] J. A. Jensen and R. Bjerngaard, "Directional velocity estimation using focusing along the flow direction: II. Experimental investigation," IEEE Trans. Ultrason., Ferroelect., Freq. Contr., vol. 50, pp. 873-880, 2003.

[14] J. A. Jensen, "Velocity vector estimation in synthetic aperture flow and B-mode imaging," in Proc. IEEE Int. Symp. Biomed. Imag. Nano to Macro, vol. 1, 2004, pp. 32-35.

[15] J. A. Jensen, O. Holm, L. J. Jensen, H. Bendsen, S. I. Nikolov, B. G. Tomov, P. Munk, M. Hansen, K. Salomonsen, J. Hansen, K. Gormsen, H. M. Pedersen, and K. L. Gammelmark, "Ultrasound research scanner for real-time synthetic aperture image acquisition," IEEE Trans. Ultrason., Ferroelect., Freq. Contr., vol. 52, no. 5, pp. 881-891, May 2005.

[16] K. V. Ramnarine, D. K. Nassiri, P. R. Hoskins, and J. Lubbers, "Validation of a new blood mimicking fluid for use in Doppler flow test objects," Ultrasound Med. Biol., vol. 24, pp. 451-459, 1998.

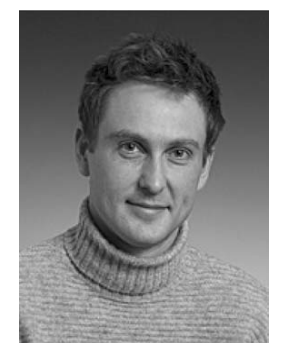

Jacob Kortbek earned his Bachelor of Science degree in electrical engineering in 1999. From 1999 until 2002 his was employed as a development engineer at Bang \& Olufsen in Denmark. He received his Master of Science degree in electrical engineering in 2004 from the Technical University of Denmark and is currently employed as an industrial Ph.D. student at B-K Medical and Ørsted $\bullet$ DTU at the Technical University of Denmark doing research in synthetic aperture ultrasound imaging.

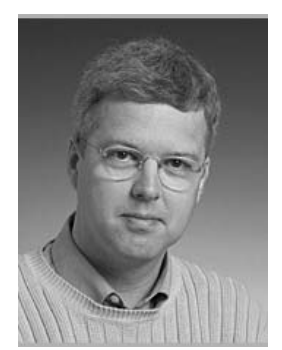

Jørgen Arendt Jensen (M'93-SM'02) earned his Master of Science degree in electrical engineering in 1985 and the Ph.D. degree in 1989, both from the Technical University of Denmark. He received the Dr.Techn. degree from the university in 1996. He has published a number of papers on signal processing and medical ultrasound and the book "Estimation of Blood Velocities Using Ultrasound", Cambridge University Press, in 1996. $\mathrm{He}$ is also the developer of the Field II simulation program. He has been a visiting scientist at Duke University, Stanford University, and the University of Illinois at Urbana-Champaign. He is currently a full professor of Biomedical Signal Processing at the Technical University of Denmark at Ørsted•DTU and head of the Center for Fast Ultrasound Imaging. He is also adjunct professor at the Faculty of Health Sciences, University of Copenhagen. He has given courses on blood velocity estimation at both Duke University and the University of Illinois and teaches biomedical signal processing and medical imaging at the Technical University of Denmark. He has given several short courses on simulation, synthetic aperture imaging, and flow estimation at international scientific conferences. He has received several awards for his research. He is also the co-organizer of a new biomedical engineering education offered by the Technical University of Denmark and the University of Copenhagen. His research is centered around simulation of ultrasound imaging, synthetic aperture imaging and blood flow estimation and constructing systems for such imaging. 